\title{
Common variants modify the age of onset for basal cell carcinomas in Gorlin syndrome
}

\author{
Binnaz Yasar ${ }^{1,2}$, Helen J Byers ${ }^{1,2}$, Miriam J Smith ${ }^{1,2}$, John Lear ${ }^{3}$, Deemesh Oudit ${ }^{4}$, Zaynab Bholah ${ }^{1}$, \\ Stephen A Roberts ${ }^{5}$, William G Newman ${ }^{1,2,6}$ and D Gareth Evans ${ }^{\star, 1,2,6}$
}

Gorlin syndrome is an autosomal dominant disorder, characterized by multiple early-onset basal cell carcinomas (BCCs) and jaw keratocysts. Through association studies in cohorts of sporadic BCC, nine genetic variants have previously been identified to increase the risk of BCC. The nine SNPs were genotyped by Taqman allelic discrimination in 125 individuals with Gorlin syndrome. Kaplan-Meier survival curves and Cox proportional-Hazard regression analysis were applied to determine the association between genotypes and age of first BCC in individuals with Gorlin syndrome. The p.(Arg151Cys) variant in MC1R (rs1805007) was associated with an earlier median age of onset of BCC of 27 years (95\% Cl: 20-34) compared with 34 years (95\% Cl: $30-40)$ for wild-type individuals (hazard ratio $(\mathrm{HR})=1.64,95 \% \mathrm{Cl}: 1.04-2.58, P=0.034$ ). The risk allele of the variant at the chromosome $5 \mathrm{p} 15$ locus encompassing TERT-CLPTM1L (rs401681) was also associated with an earlier median onset of BCC, 31 years (95\% Cl: 28-37) compared with 41 years $(95 \% \mathrm{Cl}: 32-48, \mathrm{HR}=1.44,95 \% \mathrm{Cl}: 1.08-1.93$, $\boldsymbol{P}=0.014)$. In individuals with a risk allele at either rs 1805007 or rs 401681 the median time to BCC was 31 years of age (95\% Cl: $28-34)$ compared with 44 years of age $(95 \% \mathrm{Cl}: 38-53)$ in wild-type individuals $(\mathrm{HR}=2.48,95 \% \mathrm{Cl}: 1.47-4.17$, $\boldsymbol{P}=0.0002$ ). Our findings may have implications for future personalized risk estimates and BCC screening strategies in individuals with Gorlin syndrome.

European Journal of Human Genetics (2015) 23, 708-710; doi:10.1038/ejhg.2014.167; published online 27 August 2014

\section{INTRODUCTION}

Gorlin syndrome (naevoid basal cell carcinoma syndrome) is a rare, fully penetrant autosomal dominant disorder characterized by multiple basal cell carcinomas (BCCs) and jaw keratocysts. ${ }^{1}$ It is associated with skeletal abnormalities, including bifid, extra or missing ribs, fused or wedge-shaped vertebrae and short fourth metacarpals. ${ }^{2,3}$ Distinguishing facial features associated with the syndrome include milia, macrocephaly, bossing of the forehead, coarse facial features and hypertelorism..$^{2-4}$ Palmar and plantar pits and calcification of the falx cerebri are common characteristic findings. ${ }^{2-4}$ Other significant complications include childhood medulloblastoma, ovarian and cardiac fibromas, ${ }^{2}$ ocular anomalies, and cleft lip and palate. ${ }^{4}$

One of the main defining features of Gorlin syndrome is the early onset of multiple BCCs, ${ }^{4}$ with a median age of onset of 25 years. ${ }^{5}$ The behaviour of BCCs is similar in Gorlin syndrome to that in sporadic cases in the general white European population, as they rarely metastasize and cannot be distinguished histologically. ${ }^{6,7}$

In Gorlin syndrome, the incidence and numbers of BCCs are similar in both sexes. ${ }^{4}$ At age 20 years the cumulative incidence of BCC is $14 \%$ in males and $12 \%$ in females, and by 50 years of age $77 \%$ of females and $80 \%$ of males have developed BCC. ${ }^{4}$ There is a strong association between increased BCC risk in Gorlin syndrome and both sun exposure, particularly UVB radiation, ${ }^{6}$ and skin pigmentation, where $90 \%$ of Caucasian individuals with Gorlin syndrome develop
BCCs compared with only $40 \%$ of African-Americans with the condition. ${ }^{2,4,6}$

Gorlin syndrome usually results from a pathogenic variant in the tumour suppressor PTCH1. ${ }^{8}$ Loss of PTCH1 leads to uncontrolled downstream expression in the Hedgehog pathway. ${ }^{9,10}$ Somatic variants in PTCH1 and TP53 genes are often present in sporadic BCC cases, and are considered as the main targets for UV radiationinduced variant formation. ${ }^{11}$

In addition to highly penetrant genes, common low-penetrance alleles that increase susceptibility to BCC have been identified through association studies of sporadic BCC cohorts. Variants in melanocortin 1 receptor $(M C 1 R)$ gene, a major contributor to skin pigmentation, have been associated with risk of BCCs. ${ }^{12}$ A genome-wide association study (GWAS) of over 2000 BCC cases of European ancestry in the United States replicated the strong association with the $M C 1 R$ p.(Arg151Cys) missense variant (rs1805007). ${ }^{13}$ A noncoding variant at the chromosome 6p25 locus near EXOC (rs12210050) and a variant at the 13q32 locus near UBAC2, which encodes ubiquitin-associated domain containing protein 2 (rs7335046), also reached genome-wide significance. ${ }^{13}$

An independent GWAS of over 2000 BCC cases of Icelandic and Eastern European origin reported two loci at 1 p36 (rs7538876) and 1q42 (rs801114) associated with BCC susceptibility. ${ }^{14}$ In a follow-up GWAS of over 3000 BCC cases, by the same group, further variants

${ }^{1}$ Manchester Centre for Genomic Medicine, University of Manchester, Manchester Academic Health Sciences Centre (MAHSC), Manchester, UK; ${ }^{2}$ Department of Genetic Medicine, Manchester Centre for Genomic Medicine, MAHSC, St Mary's Hospital, Central Manchester University Hospitals NHS Foundation Trust (CMFT), Manchester, UK. ${ }^{3}$ Department of Dermatology, Central Manchester University Hospitals NHS Foundation Trust (CMFT), Manchester, UK; ${ }^{4}$ Department of Surgery, The Christie NHS Foundation Trust, MAHSC, Manchester, UK; ${ }^{5}$ Centre for Biostatistics, Institute of Population Health, University of Manchester, Manchester, UK

${ }^{6}$ These authors contributed equally to this work.

*Correspondence: Professor DG Evans, Department of Genetic Medicine, Manchester Centre for Genomic Medicine, St Mary's Hospital, Hathersage Road, Manchester M13 9WL, UK. Tel: +44161 276 6002; Fax: +44 161276 6145; E-mail: gareth.evans@cmft.nhs.uk

Received 3 March 2014; revised 30 May 2014; accepted 30 May 2014; published online 27 August 2014 
Table 1 Genotype frequencies for the BCC susceptibility variants in the Gorlin syndrome cohort

\begin{tabular}{|c|c|c|c|c|c|}
\hline & Gene/locus & Homozygous for wild-type allele & Heterozygous & Homozygous for risk allele & HWE P-value \\
\hline rs1805007 $(n=113)$ & $M C 1 R$ & $85(75 \%)$ & $27(24 \%)$ & $1(1 \%)$ & 0.47 \\
\hline rs7335046 ( $n=119)$ & UBAC2 (13q32) & $85(71 \%)$ & $33(28 \%)$ & $1(1 \%)$ & 0.25 \\
\hline rs401681 $(n=118)$ & TERT-CLPTM1L (5p15) & $40(34 \%)$ & $58(49 \%)$ & $20(17 \%)$ & 0.90 \\
\hline rs12210050 $(n=115)$ & EXOC2 (6p25) & $68(59 \%)$ & 39 (34\%) & $8(7 \%)$ & 0.47 \\
\hline rs7538876 ( $n=107)$ & $1 \mathrm{p} 36$ & $48(45 \%)$ & $45(42 \%)$ & $14(13 \%)$ & 0.51 \\
\hline rs801114 $(n=101)$ & $1 q 42$ & $40(39 \%)$ & $37(37 \%)$ & $24(24 \%)$ & 0.01 \\
\hline rs2151280 ( $n=119)$ & $9 p 21$ & $45(38 \%)$ & $54(45 \%)$ & $20(17 \%)$ & 0.58 \\
\hline rs157935 $(n=120)$ & $7 q 32$ & $49(41 \%)$ & $58(48 \%)$ & $13(11 \%)$ & 0.50 \\
\hline rs11170164 $(n=120)$ & KRT5 & $94(78 \%)$ & $24(20 \%)$ & $2(2 \%)$ & 0.75 \\
\hline
\end{tabular}

including keratin 5 (KRT5, rs11170164), and the 9p21 (rs2151280), 7q32 (rs157935) and TERT-CLPTM1L (rs401681) loci were associated with an increased BCC risk. ${ }^{15}$

In this study, we genotyped nine variants previously associated with increased BCC risk, in a cohort of individuals with Gorlin syndrome, to assess their effect on the age of BCC onset.

\section{MATERIALS AND METHODS}

A register of families affected with Gorlin syndrome was established in the North-West of England in 1990. ${ }^{2}$ All individuals on the register meet the clinical criteria and/or have been found to have a pathogenic PTCH1 variant, confirming a diagnosis of Gorlin syndrome. Data were collated on the presence or absence of BCCs and age of onset of the first BCC. There were 125 individuals with Gorlin syndrome with available DNA samples for inclusion in the study.

Nine SNPs, previously published as being associated with increased BCC risk ${ }^{13-15}$ (rs1805007 chr16.hg19:89919709C > T, rs12210050 chr6.hg19:475489C > T, rs7335046 chr13.hg19:99389484G > C, rs7538876 chr1.hg19:17395867G > A, rs801114 chr1.hg19:228862088G > A, rs401681 chr5.hg19:1321972G > A, rs2151280 chr9.hg19:22034720G > A, rs157935 chr7.hg19:130900794T $>$ G and rs11170164 chr12.hg19:52913668G > T), were genotyped using pre-designed Taqman SNP genotyping allelic discrimination assays (Applied Biosystems, Warrington, UK). SNPs can be found on dbSNP (URL: http://www.ncbi.nlm.nih.gov/SNP/).

Of the genotyped samples, $8 \%$ were selected for DNA sequencing analysis for quality assurance. The Statistics Package for IBM SPSS 20 was used for statistical analysis. A dominant model was used for analyses. Kaplan-Meier survival estimates of the age of first BCC onset were generated for each genotype. Cox proportional hazard regression analysis was used to estimate hazard ratios (HRs) and the significance of genotype effects with the level of significance set at $P<0.05$.

\section{RESULTS}

A total of 125 white Northern European Gorlin syndrome patients were included, with ages ranging from 4 to 89 years. The age of BCC onset in Gorlin syndrome patients ranged from 4 to 76 years of age with a Kaplan-Meier estimated median of 33 (95\% CI: $28-37)$ years. ${ }^{2}$ There were $68(55 \%)$ female and $57(46 \%)$ male participants. All genotypes, except rs801114, were consistent with Hardy-Weinberg equilibrium $(P>0.05)$ and the allele frequencies consistent with published control dataset, as reported in the European-American data set of the Exome Variant Server (evs.gs.washington.edu/EVS) (Table 1).

The Kaplan-Meier curves demonstrate a difference between individuals either heterozygous or homozygous for the risk allele $(\mathrm{T})$ of the missense variant in MC1R (rs1805007) compared with those

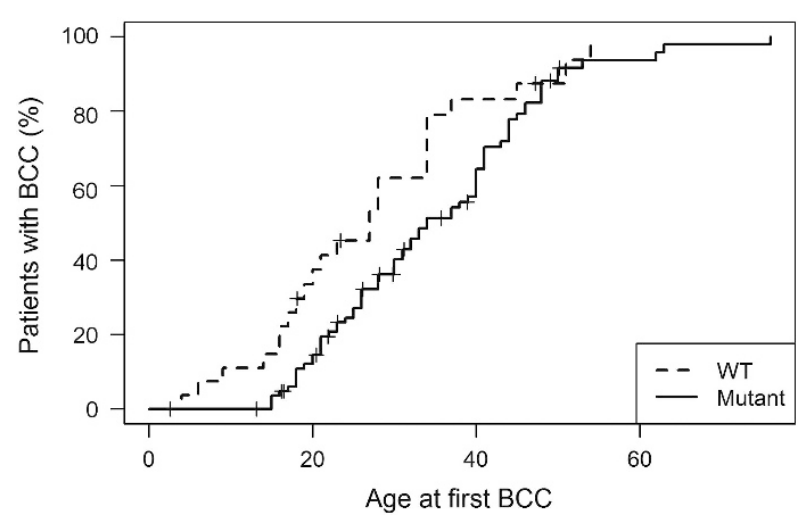

Figure 1 Kaplan-Meier curve demonstrating the effect of rs1805007 $(M C 1 R)$ genotypes on age of BCC onset in Gorlin syndrome.

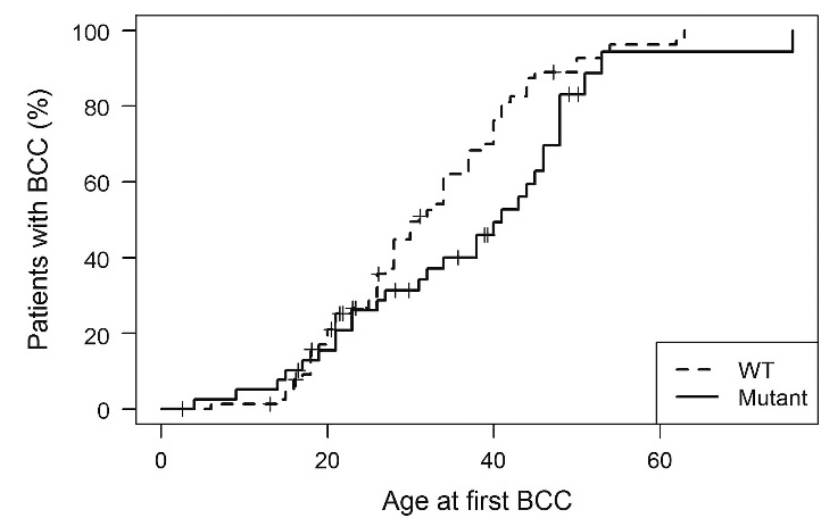

Figure 2 Kaplan-Meier curve demonstrating the effect of rs401681 (TERT-CLPTM1L) genotypes on age of BCC onset in Gorlin syndrome.

homozygous for the wild-type allele (Figure 1). Individuals with the risk allele had an earlier median onset of BCC of 27 years (95\% CI: 20-34) compared with 34 years (95\% CI: 30-40) for wild-type individuals $(\mathrm{HR}=1.64,95 \% \mathrm{CI}: 1.04-2.58, P=0.034)$.

Individuals who had one or more TERT-CLPTM1L (rs401681) risk alleles also had a significantly earlier median age of BCC onset of 31 years (95\% CI: 28-37) compared with 41 years (95\% CI: 32-48) for wild-type individuals $(\mathrm{HR}=1.44,95 \% \mathrm{CI}: 1.08-1.93, P=0.014$, Figure 2). There were no statistically significant associations between the age of onset of BCC and any of the other BCC susceptibility variants with only modest HR estimates of $0.84-1.10$ and the $95 \%$ CI excluding $>2$-fold effects (Supplementary Table S1). 
The median time to first BCC was 31 years (95\% CI: 28-34) in individuals with either a rs1805007 or a rs401681 variant and 44 years (95\% CI: $38-53$ ) in wild-type individuals ( $\mathrm{HR}=2.48$, 95\% CI: $1.47-$ $4.17, P=0.0002)$. Notably there is a significant negative interaction between the two associated variants (rs1805007 and rs401681) $(P=0.002)$, with a HR of 2.3 for patients with both variants similar to that with either variant alone.

\section{DISCUSSION}

Like many autosomal dominant conditions, Gorlin syndrome is strikingly variable in terms of clinical presentation. ${ }^{16}$ No significant genotype-phenotype correlations have been reported between the type or position of $\mathrm{PTCH} 1$ pathogenic variants and the clinical characteristics of Gorlin syndrome, including the age of BCC onset. ${ }^{2}$ This suggests that environmental factors or modifying genes influence the variable phenotype.

The present study was undertaken to investigate the effect of nine SNPs identified through previous association studies of sporadic BCC on the age of onset of BCC in individuals with Gorlin syndrome. As the variants have previously been associated with an increased risk of BCC, we did not correct for multiple testing. Two variants were significantly associated with an earlier age of BCC onset in Gorlin syndrome, a variant in the skin pigmentation gene $M C 1 R$, and a variant at the TERT-CLPTM1L locus.

The MC1R variant is associated with red hair colour and was the strongest association in the GWAS analysis of sporadic BCC. ${ }^{13}$ The increased risk is consistent with recent epidemiological data of an increased risk of BCC in individuals with red hair. ${ }^{17}$ This emphasizes that individuals with Gorlin syndrome with type 1 skin, and in particular red hair colour, need to take extra care with sun exposure to minimize their BCC risk.

Previous studies have reported the median age of BCC onset at 25 years for patients with Gorlin syndrome. ${ }^{2}$ Individuals in this study had a higher median age of BCC onset at 33 years. However, $42 \%$ of individuals with the MC1R risk allele had a BCC by the age of 20 years compared with only $17 \%$ without this variant.

The 1.64-fold increased risk associated with the MC1R variant for BCC age of onset in individuals with Gorlin syndrome is similar to that observed for the increased risk of sporadic BCC conferred by this variant. ${ }^{13}$

The variant at the TERT-CLPTM1L locus has been associated with multiple cancer types including lung, bladder and prostate cancers. ${ }^{18}$ Individuals with the variant have a propensity to shortened telomeres with increasing age. ${ }^{18}$ In a study of over 30000 cancer cases and 45000 controls, no association between the TERT-CLPTM1L and several pigment phenotypes including hair and eye colour, freckling or Fitzpatrick skin-type score was identified. ${ }^{18}$ The increased risk conferred by the TERT-CLPTM1L variant in Gorlin syndrome is comparable to that in sporadic BCC. ${ }^{15}$ The median time to BCC was reduced by over 10 years with the presence of one or both risk alleles for either variant in MC1R or at TERT-CLPTM1L.

Our findings in Gorlin syndrome share analogy with the studies of breast cancer susceptibility variants identified by GWAS in sporadic breast cancer and how these modify the risk of breast cancer in individuals with familial predisposition due to BRCA2 and, to a lesser extent, BRCA1 pathogenic variants. ${ }^{19,20}$
Only 125 individuals from 78 families (mean 1.6, range 1-6 family members) were available for consideration in this study and there may be some inflation of risks associated with the analysis of multiple members from the same family due to shared environmental factors or other genetic susceptibilities. However, there were no large families that skewed the allele frequencies.

The study provides novel evidence of the role of two BCC riskassociated variants on the age of onset of BCC in Gorlin syndrome. These findings may have implications for future personalized risk estimates and BCC screening strategies in individuals with Gorlin syndrome.

\section{CONFLICT OF INTEREST}

The authors declare no conflict of interest.

\section{ACKNOWLEDGEMENTS}

This work was supported by the British Skin Foundation and Manchester Biomedical Research Centre. DGE is a NIHR Senior Investigator.

1 Evans DG, Farndon PA: Nevoid basal cell carcinoma syndrome; 2002 Jun 20 [updated 2011 Nov 3]In: Pagon RA, Bird TD, Dolan CR eds. GeneReviews [Internet]. University of Washington, Seattle: Seattle, WA, 1993

2 Evans DGR, Ladusans EJ, Rimmer S, Burnell LD, Thakker N, Farndon PA Complications of the naevoid basal cell carcinoma syndrome: results of a population based study. J Med Genet 1993; 30: 460-464.

3 Kimonis VE, Goldstein AM, Pastakia B et al: Clinical manifestations in 105 persons with nevoid basal cell carcinoma syndrome. Am J Med Genet 1997; 69: 299-308

4 Jones EA, Sajid MI, Shenton A, Evans DG: Basal cell carcinomas in Gorlin Syndrome: a review of 202 patients. J Skin Cancer 2011; 2011: 217378.

5 Evans DG, Howard E, Giblin C et al: Birth incidence and prevalence of tumor-prone syndromes: estimates from a UK family genetic register service. Am J Med Genet 2010; 152A: 327-332.

6 Gorlin RJ: Nevoid basal cell carcinoma (Gorlin) syndrome. Genet Med 2004; 6 : 530-539

7 Tom WL, Hurley MY, Oliver DS, Shah MR, Bree AF: Features of basal cell carcinomas in basal cell nevus syndrome. Am J Med Genet 2011; 155A: 2098-2104.

8 Hahn H, Wicking C, Zaphiropoulos PG et al: Mutations of the human homolog of Drosophila patched in the nevoid basal cell carcinoma syndrome. Cell 1996; 85: 841-851.

9 Bale AE, Yu KP: The hedgehog pathway and basal cell carcinomas. Hum $\mathrm{Mol}$ Genet 2001; 10: 757-762.

10 Epstein EH: Basal cell carcinomas: attack of the hedgehog. Nat Rev Cancer 2008; 8 743-754.

11 Zhang H, Ping XL, Lee PK et al: Role of PTCH and $p 53$ genes in early-onset basal cell carcinoma. Am J Pathol 2001; 158: 381-385.

12 Scherer D, Bermejo JL, Rudnai $\mathrm{P}$ et al: MC1R variants associated susceptibility to basal cell carcinoma of skin: interaction with host factors and XRCC3 polymorphism. Int J Cancer 2008; 122: 1787-1793.

$13 \mathrm{Nan} \mathrm{H}, \mathrm{Xu} \mathrm{M}$, Kraft P et al: Genome-wide association study identifies novel alleles associated with risk of cutaneous basal cell carcinoma and squamous cell carcinoma. Hum Mol Genet 2011; 20: 3718-3724.

14 Stacey SN, Gudbjartsson DF, Sulem P et al: Common variants on $1 \mathrm{p} 36$ and $1 \mathrm{q} 42$ are associated with cutaneous basal cell carcinoma but not with melanoma or pigmentation traits. Nat Genet 2008; 40: 1313-1318.

15 Stacey SN, Sulem P, Masson G et al: New common variants affecting susceptibility to basal cell carcinoma. Nat Genet 2009; 41: 909-914.

16 Levanat S, Mubrin MK, Crnić I, Situm M, Basta-Juzbasić A: Variable expression of Gorlin syndrome may reflect complexity of the signalling pathway. Pflugers Arch 2000; 439: R31-R33

17 Kiiski V, de Vries E, Flohil SC et al: Risk factors for single and multiple basal cell carcinomas. Arch Dermatol 2010; 146: 848-855.

18 Rafnar T, Sulem P, Stacey SN et al: Sequence variants at the TERT-CLPTM1L locus associate with many cancer types. Nat Genet 2009; 41: 221-227.

19 Antoniou AC, Beesley J, McGuffog $L$ et al: Common breast cancer susceptibility alleles and the risk of breast cancer for BRCA1 and BRCA2 mutation carriers: implications for risk prediction. Cancer Res 2010; 70: 9742-9754.

20 Barnes DR, Antoniou AC: Unravelling modifiers of breast and ovarian cancer risk for BRCA1 and BRCA2 mutation carriers: update on genetic modifiers. J Intern Med 2012; 271: 331-343. 